\title{
Breaking the web of needless disability
}

Robert Aurbach

Uncommon Approach, USA. rob@uncommonapproach.com

Background: In 2012 I presented a program to the London IFDM entitled "Breaking the Web of Needless Disability". The model drew heavily from the latest neuroscientific theory, and was quite well received. This program represents further development of the ideas presented in London to encompass "resilience".

Hypothesis: 1. "Resilience" is a behaviour that is learned and unlearned like any other skill; 2. Understanding how humans think and react in compensation systems will yield valuable insight into the building of resilience to unnecessary disability.

Methods: Extension of existing literature and pilot studies examining the differences between those people who suffer injury and recover as expected and those who suffer the same sort of injury and spiral down into despair and dependency. Principal findings: "Resilience" is a specific behaviour beneficially affecting the sense of loss of control that attends injury. There are four specific "styles" of resilience. Each person uses one or more of these styles to a greater or lesser degree. By use of modified psychological inventory tools, the predominant resilience style of an individual can be ascertained, and approaches that enhance their resilience style can be utilised for either prevention or treatment.

Discussion: "Habits of thought" are established by repetition. For habits of thinking of one's self as able or disabled, the most significant form of repetition is internal dialog. In resilient people, there are four discrete kinds of dialog that are used to overcome a sense of loss of control. By identifying and bolstering those styles of resilience an injured person can become less susceptible to a sense of loss of control that gives rise to unnecessary disability.

Conclusion: The approach is useful in understanding failure to recover as expected, and shows promise for effective individualised treatment for injury management. 